Case Report

\title{
Multivessel Spontaneous Coronary Artery Dissection in an Unlikely Patient
}

\author{
Waqas Jehangir, ${ }^{1}$ Tarek Aly, ${ }^{2}$ Kebir H. Bedran, ${ }^{1}$ Abdalla Yousif, ${ }^{1}$ and Mark L. Niemiera ${ }^{1}$ \\ ${ }^{1}$ Raritan Bay Medical Center, Perth Amboy, NJ 08861, USA \\ ${ }^{2}$ Ross University School of Medicine, Portsmouth, Dominica \\ Correspondence should be addressed to Waqas Jehangir; wjehangir@hotmail.com
}

Received 24 October 2014; Revised 16 March 2015; Accepted 17 March 2015

Academic Editor: Tayfun Sahin

Copyright (C) 2015 Waqas Jehangir et al. This is an open access article distributed under the Creative Commons Attribution License, which permits unrestricted use, distribution, and reproduction in any medium, provided the original work is properly cited.

\begin{abstract}
When approaching the symptom of acute onset chest pain in a previously healthy 26 -year-old male, anchoring heuristic presents a challenge to healthcare workers. This diagnostic error is the healthcare professional's tendency to rely on a previous diagnosis, and, in situations where a set of symptoms might mask a rare and deadly condition, this error can prove fatal for the patient. One such condition, Spontaneous Coronary Artery Dissection (SCAD), is an uncommon and malefic presentation of coronary artery disease that can lead to myocardial infarction and sudden death. We present a case of SCAD in an otherwise healthy 26 year-old male who had been experiencing chest pain during and after sports activity. In the young, athletic male with SCAD, the danger of diagnostic error was a reality due to the broad symptomatology and the betraying demographics.
\end{abstract}

\section{Introduction}

Spontaneous Coronary Artery Dissection is a deadly and rare presentation of coronary artery disease that can lead to myocardial infarction and sudden death. As it is uncommon in young males, the recognition of SCAD in such an individual given nonspecific symptoms can mask the diagnosis at a stage where timely intervention could be lifesaving.

\section{Case Presentation}

While playing cricket, a 26-year-old Indian Asian male without any significant past medical history experienced a two-week history of intermittent, retrosternal stabbing chest pain that radiated to his left arm and shoulder. The pain was pleuritic, positional, and associated with diaphoresis and nausea. The patient was diagnosed with costochondritis by his primary care physician. Seven days prior to admission, the patient had an upper respiratory tract infection with fever and chills. He denied smoking, drinking alcohol, or to using drugs; however, he reported that he had been drinking protein shakes for 3 years for muscle building. Upon physical exam, the patient was afebrile with a $\mathrm{BP}$ of $118 / 86 \mathrm{mmHg}$,
PR of $111 / \mathrm{min}$, and RR of $22 / \mathrm{min}$. The remainder of the physical exam was completely unremarkable. Laboratory data showed Hb $15.6 \mathrm{~g} / \mathrm{dL}$, Hct 44.6, WBC $19.9 \mathrm{~K} / \mu \mathrm{L}$, platelet count $675 \mathrm{~K} / \mu \mathrm{L}$, glucose $157 \mathrm{mg} / \mathrm{dL}$, BUN $21 \mathrm{mg} / \mathrm{dL}, \mathrm{Cr} 1.2 \mathrm{mg} / \mathrm{dL}$, Ca $9.7 \mathrm{mg} / \mathrm{dL}$, albumin $4.7 \mathrm{~g} / \mathrm{dL}$, total protein $7.8 \mathrm{~g} / \mathrm{dL}$, sodium $137 \mathrm{mmol} / \mathrm{L}$, potassium $3.3 \mathrm{mmol} / \mathrm{L}$, chloride $98 \mathrm{mmol} / \mathrm{L}$, and bicarbonate $20 \mathrm{mmol} / \mathrm{L}$. Urine drug screen was negative. ECG showed ST elevation in the anterolateral leads (Figure 1). After consulting cardiology, the patient was taken to the cardiac catheterization lab for rescue angioplasty. A 95\% midspiral right coronary artery dissection with total left anterior descending spontaneous dissection was demonstrated (Figures 2 and 3). Both the dissections were classified as type 1 . The remaining vessels were normal. Left ventricular ejection fraction was measured at $15-20 \%$. Percutaneous coronary intervention (PCI) to LAD and mid-RCA was performed. Vasculitis work-up was subsequently negative.

\section{Discussion}

Spontaneous Coronary Artery Dissection (SCAD) is a rare condition that can lead to myocardial infarction, cardiogenic shock, and sudden death. SCAD is often seen in the absence 


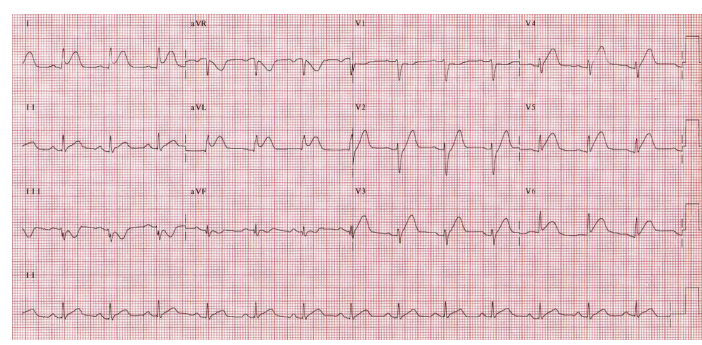

FIGURE 1: ECG showing ST elevation in the anterolateral leads.

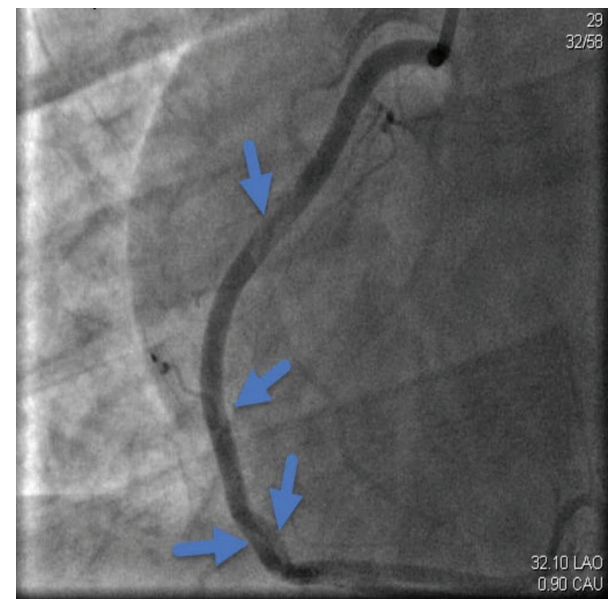

FIGURE 2: Showing 95\% mid-spiral right coronary artery dissection.

of atherosclerotic disease and is a well-recognized cause of acute coronary syndromes [1]. The disease is much more common in women than in men (58-79\% female), has a mean age of 41, carries an annual incidence of 0.26 cases per 100,000 , and has a prevalence of $0.07-0.28 \%$ [2]. With that stated, the presentation in a 26 -year-old athletic male is a rare presentation of an already rare disease.

The pathology of SCAD entails the development of a vessel wall lesion leading to a "false lumen" and flattening of the true lumen. SCAD generally outlines an intimalmedial tear that produces a communication between the vessel lumen and intramural hematoma (the false lumen) [2]. The blood that comes into the false lumen clots and separates the detached tissue. As the blood rushes into that false lumen, the dissection occurs as the separation between the intima, media, or outer media leading to a new hollow lumen that is known as the false lumen. The false and true lumens create the typical angiographic sign of coronary dissection known as "double lumen."

One of the dangerous aspects of SCAD involves its clinical presentation. It may present as the entire spectrum of coronary syndromes ranging from an asymptomatic, stable angina, NSTEMI, or STEMI to sudden cardiac death with 91\% of patients complaining of chest discomfort [2]. In addition, almost all SCAD patients have normal blood cell counts, hemoglobin, coagulation, acute phase reactants, antibodies, and hormones. To make its ambiguity an even more challenging problem, SCAD is mostly an incidental finding during

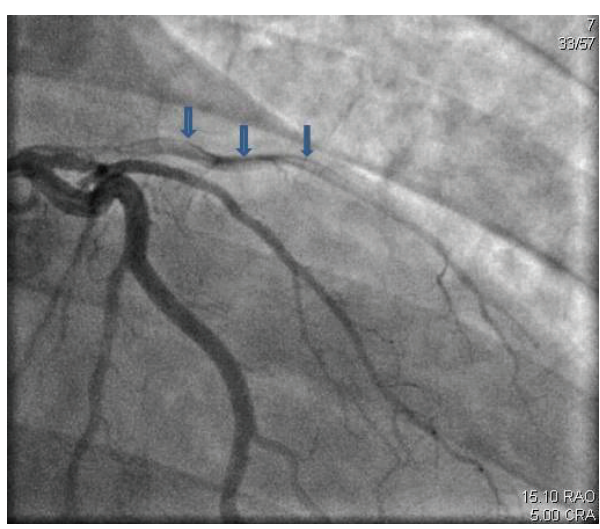

FIGURE 3: Showing total left anterior descending spontaneous dissection.

coronary angiography following STEMI or NSTEMI [2]. It is valuable to note that our patient only reported a stabbing pleuritic chest pain with left arm radiation, nausea, and diaphoresis encompassing the symptomatology of coronary syndromes in general.

SCAD has associations with atherosclerotic coronary artery disease, aortic dissection, blunt force trauma, cystic medial necrosis, and the Ehlers-Danlos syndrome; however some cases are idiopathic [3]. An association between SCAD and fibromuscular dysplasia, Marfan's syndrome, Kawasaki's disease, alpha-1 antitrypsin deficiency, and diabetic ketoacidosis has also been shown in recent studies [4]. While there are multiple connections between SCAD and the conditions listed, our patient had no pertinent medical history and thus falls into the idiopathic SCAD category.

An important facet in this case is the initial diagnosis of costochondritis made by the patient's primary care provider. The anchoring heuristic is an original diagnostic formulation that tends to influence future judgments and affects adjustments made to therapeutic plan [5]. It is of the utmost importance always not to "anchor" by demographics on the likelihood for an event to occur. Despite demographics and a decreased likelihood in a young individual, chest pain should always be taken seriously and ACS/MI should be excluded.

\section{Conflict of Interests}

The authors declare that there is no conflict of interests regarding the publication of this paper.

\section{References}

[1] K. Aghasadeghi and A. Aslani, "Spontaneous coronary artery dissection in a professional body builder," International Journal of Cardiology, vol. 130, no. 3, pp. e119-e120, 2008.

[2] D. Giacoppo, D. Capodanno, G. Dangas, and C. Tamburino, "Spontaneous coronary artery dissection," International Journal of Cardiology, vol. 175, no. 1, pp. 8-20, 2014.

[3] S. Harikrishnan, V. K. Ajithkumar, and J. M. Tharakan, "Spontaneous coronary artery dissection of all major coronary arteries," Canadian Journal of Cardiology, vol. 23, no. 4, pp. 313-314, 2007. 
[4] B. Tashtoush, A. Balagadde, and M. Bhatt, "Spontaneous coronary artery dissection with multiple coronary artery aneurysms in a patient with diabetic ketoacidosis," Journal of Thoracic Disease, vol. 6, article 5, 2014.

[5] N. Epley and T. Gilovich, "When effortful thinking influences judgmental anchoring: differential effects of forewarning and incentives on self-generated and externally provided anchors," Journal of Behavioral Decision Making, vol. 18, no. 3, pp. 199-212, 2005. 


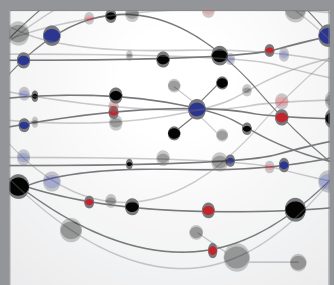

The Scientific World Journal
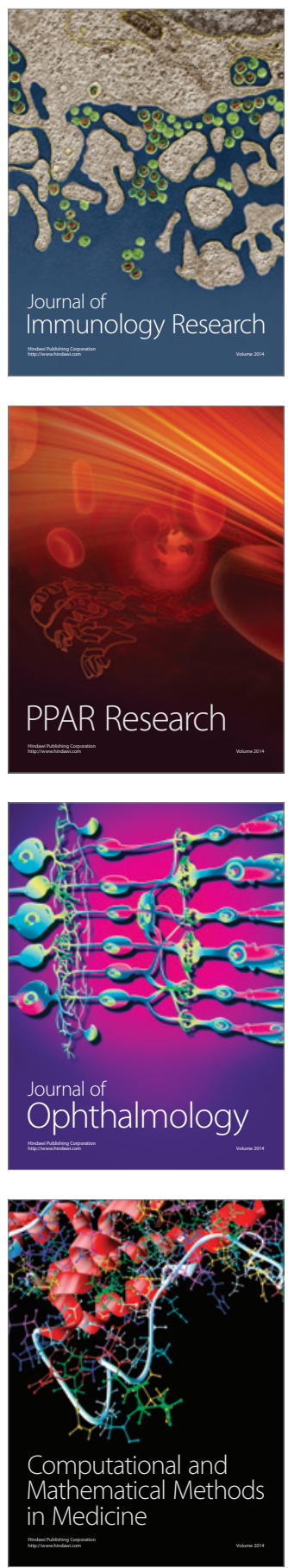

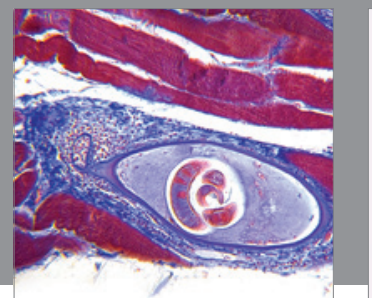

Gastroenterology

Research and Practice
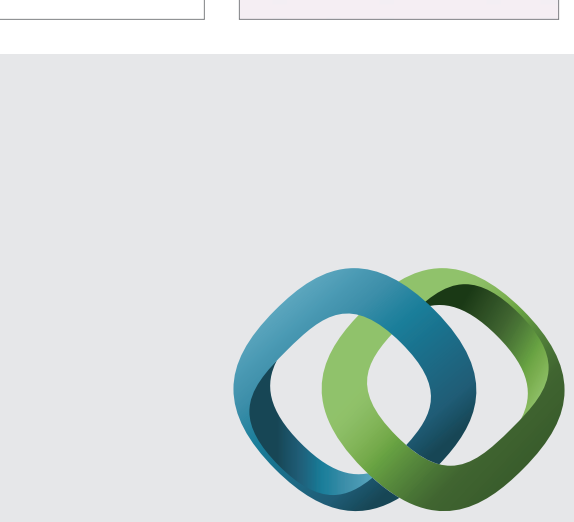

\section{Hindawi}

Submit your manuscripts at

http://www.hindawi.com
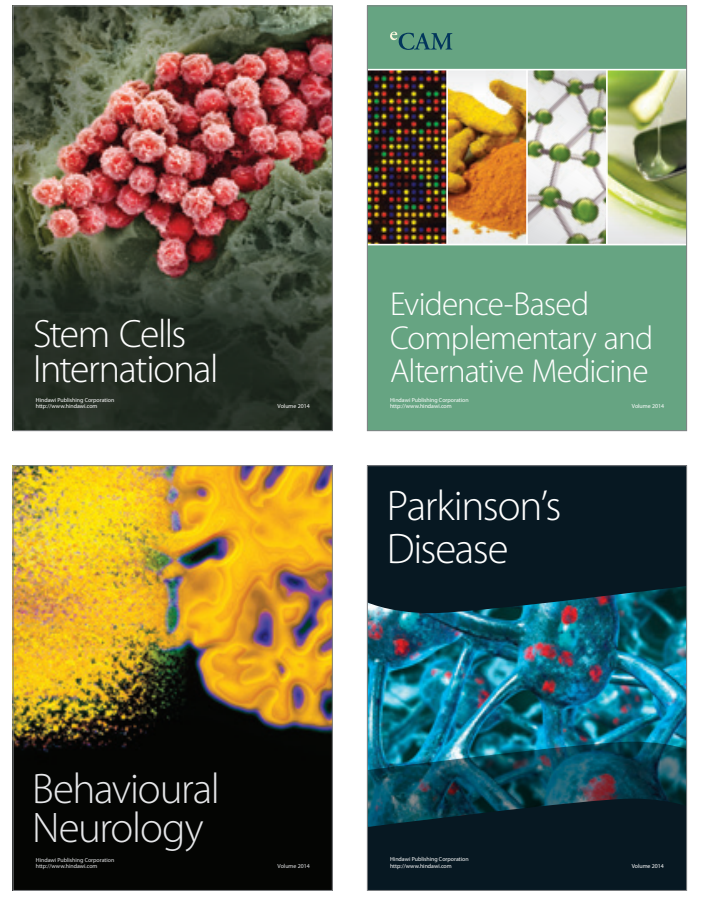
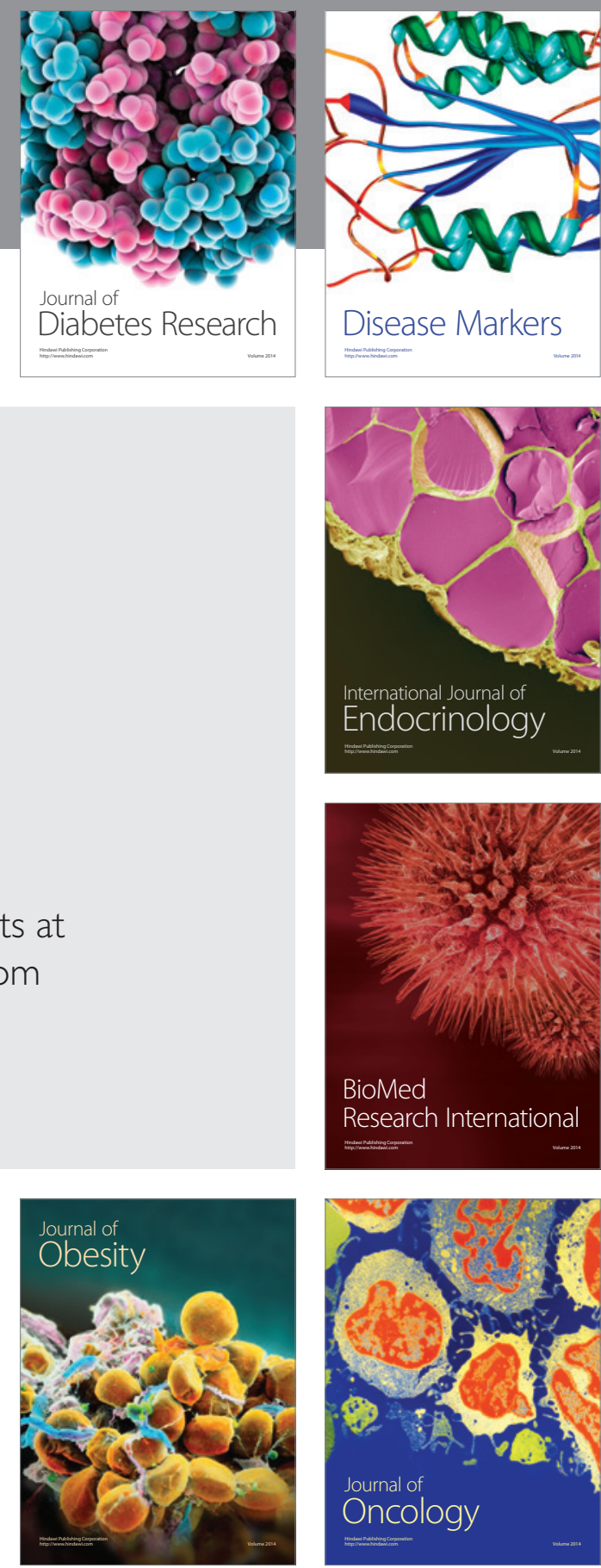

Disease Markers
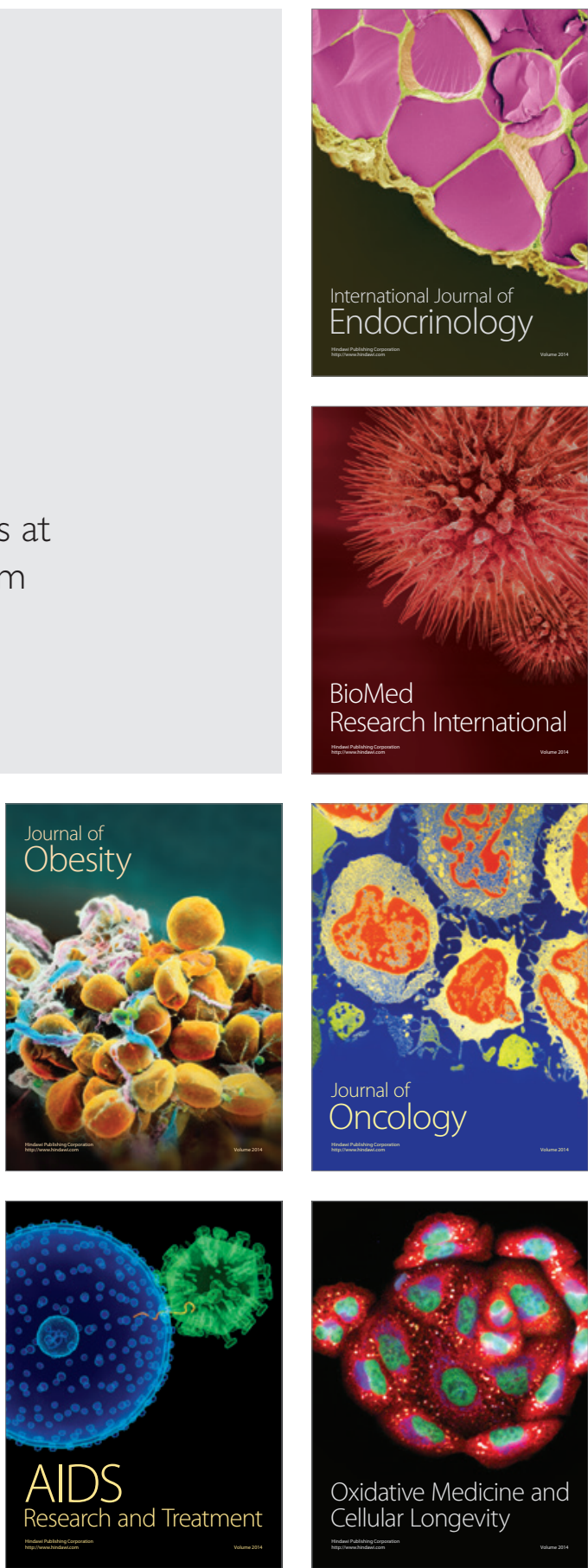